\title{
Inconsistent rheological behaviour of starch-based dysphagia products
}

\author{
C. Payne ${ }^{1}$, A. Bell ${ }^{1}$, L. Methven ${ }^{1}$ and C. Fairfield ${ }^{2}$ \\ ${ }^{1}$ Department of Food Biosciences, University of Reading, Whiteknights, Reading, Berkshire RG5 6AP, UK and ${ }^{2}$ Department \\ of Clinical Linguistics, University of Reading, Whiteknights, Reading, Berkshire RG5 6AP, UK
}

Starch-based thickeners may be given to individuals with dysphagia to favourably alter variables of the swallow reflex ${ }^{(1)}$. These are prepared to different consistencies (syrup, custard and pudding), which correspond to different viscosity levels at a shear rate of $501 / \mathrm{s}$, which is thought to represent "the average" shear rate operating in the mouth ${ }^{(2-5)}$. The viscosity ranges were established by the American Dietetic Association for the National Dysphagia Diet task force (NDD) ${ }^{(6)}$. Starch-based pre-made beverages and instant thickeners were found to be shear thinning and to exhibit great variation in consistency, especially at lower shear rates as shown by simple viscosity and the rheological profile parameters complex $\left(G^{*}\right)$, viscous $\left(G^{\prime \prime}\right)$ and elastic modulus $\left(G^{\prime}\right)$ over a range of physiological shear rates denoting the variation in structure and behaviour. At low shear rates, the large range of viscosity values for "Custard consistency" samples was found to be significant at $P<0.0001(F=77.56)$. At a shear rate of $501 / \mathrm{s}$, the viscosity was reduced considerably and the range narrowed to allow all samples to fall within the NDD guidelines. However, the variation was still found to be significant at $P<0.0001(F=98.21)$ and was largely attributed to the considerable differences between the pre-thickened instantly thickened beverages.

\begin{tabular}{lcccc}
\hline Viscosity Pa S 'custard' consistency & $\begin{array}{c}\text { Thickening agent } \\
\text { A (batch 1) }\end{array}$ & $\begin{array}{c}\text { Thickening agent } \\
\text { A (batch 2) }\end{array}$ & $\begin{array}{c}\text { Pre-thickened } \\
\text { beverage A }\end{array}$ & $\begin{array}{c}\text { Thickening } \\
\text { agent B }\end{array}$ \\
\hline At shear rate $0.11 / \mathrm{s}$ & $74.41 \pm 6.35$ & $\begin{array}{c}\text { Pre-thickened } \\
\text { beverage B }\end{array}$ \\
At shear rate $501 / \mathrm{s}$ & $1.44 \pm 0.13$ & $1.09 \pm 17.7$ & $25.97 \pm 1.77$ & $86.79 \pm 12.26$ \\
$0.9 \pm 0.01$ & $32.62 \pm 6.84$ \\
$1.46 \pm 0.1$ & $1.34 \pm 0.04$ \\
\hline
\end{tabular}

The rheological profile parameters $\left(G^{*}, G^{\prime}\right.$ and $\left.G^{\prime \prime}\right)$ showed that all samples were found to have the characteristics of a weak viscoelastic "gel" network ${ }^{(7)}$. This was shown to be much weaker for the pre-thickened beverages denoting that they have a much looser structure and thus different behaviour. Large variations in viscosity values and rheological parameters were not only evident between premade and instantly thickened beverages but also between different batches of the same products.

The 50 1/s shear rate chosen to classify the viscosity of the different consistency classes was originally based on studies from the late 1960 s, where sensory panels were used to correlate perception of viscosity with actual viscosity measurements. Later investigations showed the range varied between about 1-1000 1/s, and in fact a shear rate of about $10 \mathrm{1} / \mathrm{s}$ was identified as a more "realistic" shear rate ${ }^{(2-5)}$. These studies used healthy volunteers, usually staff and students, who were likely to be capable of developing higher shear rates than older, more fragile dysphagic patients. A recent study looking at computer simulation of pharyngeal transport of starch-thickened materials found that the shear rates generated were as low as $0.0011 / \mathrm{s}$.

Therefore, if a shear rate of $50 \mathrm{1} / \mathrm{s}$ is generated and complete destruction of the samples is attained in the mouth, then all the products fall within the NDD guidelines, and patients are receiving what they have been assessed to receive. However, if the shear rate generated is lower, as seems reasonable, and the ability to manipulate boluses of different viscoelastic structures is considered, then patients may be receiving products with very variable characteristics from what has been regarded as "standard preparation".

1. Dantas RO et al. (1990) Am J Physiol 258, G675-G681.

2. Dickie AM \& Kokini JL (1983) J Food Sci 48, 57-65.

3. Shama F et al. (1973a) J Texture Stud 4, 104-110.

4. Shama F et al. (1973b) J Texture Stud 4, 111-118.

5. Cutler AN et al. (1983) J Texture Stud 14, 377-395.

6. National Dysphagia Diet Taskforce (2002) Chicago, IL: American Dietetic Association. National Dysphagia Diet: Standardisation for Optimal Care.

7. Ferry JD (1980) Viscoelastic Properties of Polymers. 3rd ed. New York: Wiley. 